fined on the real line $X$ is an example of a function which is locally an $\epsilon$-mapping in the narrow sense, but which is not a polynomial mapping. In this case we have $f(X) \neq X$.

REMARK 2. From Theorem 3 it follows that if $F$ satisfies the assumptions of Theorem 3 , then there exists a point $x$ such that $F(x)=x$ (i.e., $F: X \rightarrow X$ has a fixed point).

\title{
REFERENCES
}

1. M. Altman, On a theorem of K. Borsuk, Bull. Acad. Polon. Sci. Cl. III vol. 5 (1957) pp. 1037-1040.

2. K. Borsuk, Ueber stetige Abbildungen der Euklidischen Raeume, Fund. Math. vol. 21 (1933) pp. 236-243.

3. F. Hausdorff, Grundzuege der Mengenlehre, New York, Chelsea, 1949.

4. C. Kuratowski, Topologie. II, Warszawa, Monografie Matematyczne, 1952.

5. M. Reichbach, Generalizations of the fundamental theorem of algebra, Bull. Res. Council Israel vol. 7 (1958) pp. 155-164.

6. - Some theorems on mappings onto, Pacific J. Math. vol. 10 (1960) pp. 1397-1407.

Technion-Israel Institute of Technology, Haifa, Israel

\section{A CHAINABLE CONTINUUM NO TWO OF WHOSE NONDEGENERATE SUBCONTINUA ARE HOMEOMORPHIC}

\section{JAMES J. ANDREWS}

R. D. Anderson and Gustave Choquet [1] gave an example of a plane continuum no two of whose nondegenerate subcontinua are homeomorphic. The object of this note is to point out that there is a chainable continuum having this property. The only change we make in the construction given in [1] is to replace the $n$-ods used by R. D. Anderson and Gustave Choquet by chainable continua $C_{n-2}$.

A subcontinuum $Y$ of a continuum $X$ is a separating continuum of $X$ if $X-Y$ is not connected and $\mathrm{Cl}(X-Y)=X$. A subcontinuum $Y$ of a continuum $X$ is a strong separating continuum if:

(1) $Y$ is a separating continuum of $X$,

(2) $X-Y$ has two components, $X_{1}$ and $X_{2}$,

(3) there are points $y_{1}, y_{2} \in Y$ such that $y_{i} \notin \mathrm{Cl}\left(X_{i}\right)$.

Let $V=\{(x, y) / y=|x|$ and $-1<x<1\}$. Let $C_{n}$ be formed from $n$ copies of $V$ and $n+1$ "lines" so that each $V$ is a strong separating continuum of $C_{n}$ as in Figure 1 (for $n=2$ ).

Received by the editors May 10, 1960. 


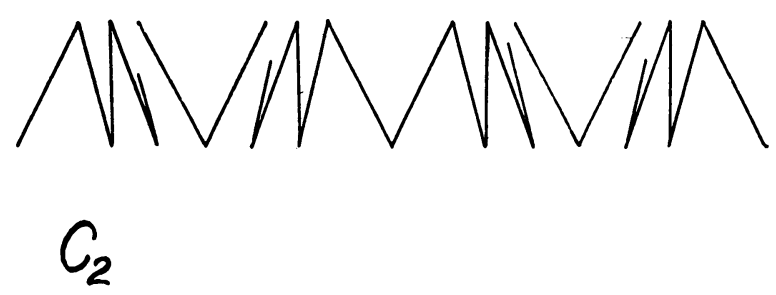

FIGURE 1

Following [1] we construct a continuum $X$ using $C_{n}$ in place of the $(n+2)$-ods used in [1]. As in [1], if $A$ and $B$ are subcontinua of $X$ we may assume $A-B$ is not empty and hence $A-B$ contains a separating continuum $Y$ of $A$. Now $Y$ contains $k$ strong separating continua. No subcontinuum of $B$ has this property. Hence $A$ and $B$ are not homeomorphic.

In order to show that $X$ is chainable we first note that each $M_{i}$ is chainable.

By Lemma 2 of [1] we have the following: For each number $\epsilon>0$ there is an integer $i$ such that $D\left(\tilde{f}_{i}^{-1}(p)\right)<\epsilon$, for $p \in M_{i}$ and $\tilde{f}_{i}$ the map induced by $f_{j}: M_{j+1} \rightarrow M_{j}$ of $X$ onto $M_{i}$. This implies that there is a $\delta>0$ such that if $U$ is open in $M_{i}$ and $D(U)<\delta$, then $D\left(\tilde{f}_{i}^{-1}(U)\right)$ $<\epsilon$.

One need only assume the contrary. Then for each $\delta>0$ there is a $U(\delta)$ such that $D(U(\delta))<\delta$ and $D\left(\tilde{f}_{i}^{-1}(U(\delta))\right)>\epsilon$. Let $p$ be a limit point of a set $P$ of points $p_{j} \in U(1 / j)$. If $U$ is an open set containing $p$ then $U \supset U(1 / j)$ for some $j$ and hence $D\left(\tilde{f}_{i}^{-1}(p)\right)>\epsilon$. But this contradicts our choice of $M_{i}$. In order to $\epsilon$-chain $X$ we need only $\delta$-chain $M_{i}$.

\section{REFERENCES}

1. R. D. Anderson and Gustave Choquet, $A$ plane continuum no two of whose subcontinua are homeomorphic, Proc. Amer. Math. Soc. vol. 10 (1960) pp. 347-353.

UNIVERSITY OF WISCONSIN 